\title{
Polymer solar cells with gold nanoclusters decorated multi-layer graphene as transparent electrode
}

\author{
Di Zhang, ${ }^{1}$ Wallace C. H. Choy, ${ }^{1, a)}$ Charlie C. D. Wang, ${ }^{1}$ Xiao Li, ${ }^{2}$ Lili Fan, ${ }^{2}$ Kunlin Wang, ${ }^{2}$ \\ and Hongwei Zhu ${ }^{2, b), c)}$ \\ ${ }^{1}$ Department of Electrical and Electronic Engineering, The University of Hong Kong, Pokfulam Road, \\ Hong Kong, China \\ ${ }^{2}$ Key Laboratory for Advanced Manufacturing by Materials Processing Technology, Department \\ of Mechanical Engineering, Tsinghua University, Beijing 100084, China
}

(Received 22 September 2011; accepted 7 November 2011; published online 30 November 2011)

\begin{abstract}
A thin layer of ultraviolet-ozone (UVO) treated gold $(\mathrm{Au})$ is introduced on multi-layer graphene (MLG) to enable the MLG as an effective anode for polymer solar cells (PSCs). By optimizing the $\mathrm{Au}$ thickness and the durations of the UVO treatments at different stages, MLG PSCs with enhanced fill factor and power conversion efficiency are obtained, exhibiting better performance compared with MLG devices directly modified with UVO and poly(3,4-ethylenedioythiophene): poly(styrenesulfonate). Further analysis shows that UVO treated Au provides favorable band alignment at the MLG/polymer interface. Moreover, the improved interfacial contact and shortened UVO durations reduce the series resistance of PSCs significantly. (C) 2011 American Institute of Physics. [doi:10.1063/1.3664120]
\end{abstract}

Polymer solar cells (PSCs) have been considered as a promising candidate for future photovoltaic applications due to the mechanical and chemical flexibility of the organic materials, as well as the low-cost processing methods which enable large area applications. ${ }^{1}$ One essential aspect of PSCs is the transparent electrode, which must be electrically conductive while retaining excellent transparency. More importantly, the work function (WF) of the electrodes should be tuned in accordance with the molecular orbital of the donor or the acceptor in order to minimize injection barrier at anode or cathode, respectively. Organic photovoltaics commonly utilize indium tin oxide (ITO) as bottom anode, which is relatively expensive ${ }^{2}$ and chemically unstable. ${ }^{3}$ Moreover, ITO is rather brittle, ${ }^{4}$ which is not inherently compatible with the flexibility nature of organic materials.

Recently, graphene has attracted extensive research interest for next generation transparent electrodes due to its uniformly high transparency, excellent electrical conductivity, and low synthesis cost. ${ }^{5}$ In addition, it has been demonstrated that graphene is more mechanically robust than ITO, showing superior performance under bending. ${ }^{6}$ However, one of the critical issues is the tuning of WF. In conventional poly(3hexylthiophene):[6,6]-phenyl C61-butyric acid methyl ester (P3HT:PCBM)-based PSCs, a large hole injection barrier is formed between graphene anode and the donor material P3HT. Poly(3,4-ethylenedioythiophene):poly(styrenesulfonate) (PEDOT:PSS) has been used as the hole injection layer (HIL), and ultraviolet-ozone (UVO) treatment on graphene has been reported to improve the wettability and uniform coverage of PEDOT:PSS. ${ }^{7,8}$ However, oxygen groups introduced by UVO treatment tend to disrupt the aromatic structures of graphene, resulting in rapid increase in the series

\footnotetext{
${ }^{a)}$ Electronic mail: chchoy@eee.hku.hk.

${ }^{b)}$ Present address: Center for Nano and Micro Mechanics, Tsinghua University, Beijing 100084, China.

${ }^{c}$ Electronic mail: hongweizhu@tsinghua.edu.cn.
}

resistance $\left(R_{s}\right)$ of PSCs. ${ }^{7,8}$ Therefore, research efforts are directed towards modifications rendering graphene compatible with PEDOT:PSS by noncovalent functionalization of graphene, ${ }^{7}$ synthesizing hydrophobic PEDOT, ${ }^{8}$ or adding an extra layer. ${ }^{2,9}$ Yet, there are concerns in using PEDOT:PSS due to its strongly acidic $(\mathrm{pH} \sim 1)$ aqueous suspensions ${ }^{10}$ and the potential introduction of water into the active layer. ${ }^{11}$ While many materials have been reported as alternative HILs for ITO, ${ }^{12-14}$ only very recently, a few have been considered as HIL for graphene. ${ }^{15}$

In this work, we focus on introducing an alternative approach to realize transparent graphene anodes. Based on earlier work, ${ }^{16}$ we report the use of very thin thermally evaporated gold $\mathrm{Au}$ ) nanoclusters with proper UVO treatments to facilitate efficient hole collection at graphene electrodes, which significantly benefits device performance while avoiding issues arising from PEDOT:PSS. We will investigate the effects of Au thickness and UVO treatments for optimizing device performance. Ultraviolet photoemission spectroscopy (UPS) is conducted to further analyze the WF shift at the graphene/polymer interface modified by UVO-treated Au.

Multi-layer graphene (MLG) films were synthesized on copper foils by a chemical vapor deposition process. ${ }^{17,18}$ Sheet resistance (SR) of 1.2-2.2 $\mathrm{k} \Omega \mathrm{sq}^{-1}$ and average transmittance of $75-82 \%$ of the MLG were characterized by a fourpoint probe and spectroscopic ellipsometry, respectively.

The MLG samples were treated with UVO (pre-UVO) and then transferred to vacuum chamber immediately for $\mathrm{Au}$ evaporation. The MLG/Au samples were treated by another UVO treatment (post-UVO) again. Meanwhile, MLG/ PEDOT samples were prepared for comparison, with PEDOT:PSS (Baytron AI 4083) directly spin-coated on MLG samples, followed by heating at $140^{\circ} \mathrm{C}$ for $10 \mathrm{~min}$. While for MLG/PEDOT devices with UVO, MLG films were treated by UVO before PEDOT:PSS spin-coating. The prepared MLG samples were then transferred into a 
nitrogen-filled glove box for spin-coating the blend of P3HT and PCBM with $1: 1$ weight ratio $(20 \mathrm{mg} / \mathrm{mL}$ each dissolved in 1,2-dichlorobenzene). Before annealing at $110^{\circ} \mathrm{C}$ for $10 \mathrm{~min}$ on a hotplate, solvent annealing was utilized as described elsewhere. ${ }^{19} \mathrm{LiF}(1 \mathrm{~nm}) / \mathrm{Al}$ was thermally evaporated as the top cathode, which defines the device area as 0.03-0.05 $\mathrm{cm}^{2}$. For reference, PEDOT-based ITO device with the structure of ITO/PEDOT:PSS/P3HT:PCBM/LiF/Al was also fabricated. Current density $(J)$-voltage $(V)$ characteristics were measured by using a Keithley 2635 sourcemeter and ABET AM $1.5 \mathrm{G}$ solar simulator. ${ }^{20}$

Four samples including A: pristine MLG, B: MLG/preUVO (3.5 min)/Au ( $2 \mathrm{~nm})$ without post-UVO, C: MLG/preUVO $(3.5 \mathrm{~min}) / \mathrm{Au}(2 \mathrm{~nm})$ with $2 \mathrm{~min}$ post-UVO, and $\mathbf{D}$ : MLG/pre-UVO $(3.5 \mathrm{~min}) / \mathrm{Au}(2 \mathrm{~nm})$ with $6 \mathrm{~min}$ post-UVO were prepared for UPS measurement using a He discharged lamp (He I $21.22 \mathrm{eV}$, Kratos Analytical).

Fig. 1 shows the $J-V$ characteristics and performance of a representative set of PSCs. Conventional ITO/PEDOT:PSS/ $\mathrm{P} 3 \mathrm{HT}: \mathrm{PCBM} / \mathrm{LiF} / \mathrm{Al} \mathrm{PSC}$ is also listed for reference. $R_{S}$ is calculated from the inverse slope of the illuminated $J-V$ characteristics at $1.0 \mathrm{~V}$. For pristine MLG PSCs, no photovoltaic behavior is observed. While the introduction of PEDOT:PSS between MLG and polymer yields limited photovoltaic performance, conformal coating of aqueous PEDOT:PSS on the hydrophobic surface of graphene remains a challenging issue. The uniformity of PEDOT:PSS on MLG films is improved by applying UVO treatment in advance to convert the hydrophobic graphene surfaces into hydrophilic ones. ${ }^{7,8}$ MLG/ PEDOT:PSS PSC with 8 min of UVO treatment demonstrates the best power conversion efficiency (PCE) $(0.59 \%)$, with high open-circuit voltage $\left(V_{O C}\right)$ and increased short-circuit current density $\left(J_{S C}\right)$, yet, fill factor $(\mathrm{FF})$ remains low. As stated such a treatment has negative impact on the conductivity of MLG, which gives rise to $R_{S}$ increasing from $32.9 \Omega$ $\mathrm{cm}^{2}$ to $114.3 \Omega \mathrm{cm}^{2}$. While $J_{S C}$ and $V_{O C}$ are initially enhanced by a short period of UVO treatment, such benefits are outweighed by the loss in conductivity when the treatment time is beyond the optimal value.

In this regard, we propose that a thin layer of $\mathrm{Au}$ with two separate short UVO treatments (pre-UVO and post-

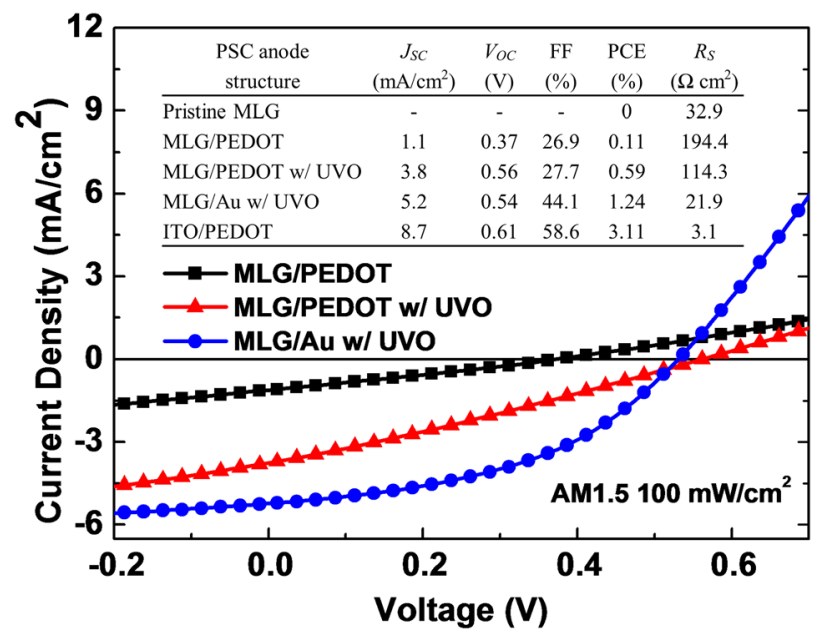

FIG. 1. (Color online) Illuminated $J-V$ characteristics of representative MLG PSCs. Inset shows the performance of respective MLG PSCs and reference ITO/PEDOT:PSS/P3HT:PCBM/LiF/Al PSC.
UVO) can enable graphene as an effective anode in PSCs by modifying the interface between graphene and active layer while retaining its conductivity. As a result, $\mathrm{FF}$ and $J_{S C}$ are significantly enhanced due to notable reduction in $R_{S}$, and $V_{O C}$ is largely improved owing to the WF tuning by UVOtreated $\mathrm{Au}$. When a process of pre-UVO/Au evaporation/ post-UVO of $3.5 \mathrm{~min} / 2 \mathrm{~nm} / 2 \mathrm{~min}$ is utilized, the following PSC characteristics are obtained: high $V_{O C}$ of $0.52-0.54 \mathrm{~V}$, increased $J_{S C}$ of $4.5-5.5 \mathrm{~mA} / \mathrm{cm}^{2}$, a greatly enhanced FF of $43-48 \%$, and a best PCE of $1.24 \%$, which is significantly better than that of MLG devices directly modified with UVO and PEDOT:PSS. In addition, the resistivity of MLG increases only by $40-50 \%$ (to $2-3 \mathrm{k} \Omega \mathrm{sq}^{-1}$ ), achieving smaller increment compared with MLG treated by $8 \mathrm{~min}$ UVO for PEDOT:PSS spin-coating $\left(>4 \mathrm{k} \Omega \mathrm{sq}^{-1}\right)$.

Our results show that pre-UVO treatment favors $\mathrm{Au}$ adhesiveness on MLG films and greatly benefits $R_{S}$ (see Table I). Indeed, when no pre-UVO is applied, the device behaves similar to pristine MLG device. When pre-UVO is increased from $1 \mathrm{~min}$ to $3.5 \mathrm{~min}, R_{S}$ decreases from $67.2 \Omega$ $\mathrm{cm}^{2}$ to $21.9 \Omega \mathrm{cm}^{2}$ indicating improved interfacial contact. As a result, the devices show remarkable improvement over $J_{S C}$ (from $3.4 \mathrm{~mA} / \mathrm{cm}^{2}$ to $5.2 \mathrm{~mA} / \mathrm{cm}^{2}$ ) and FF (from $31.2 \%$ to $44.1 \%$ ). Beyond the peak of performance at a treatment time of $3.5 \mathrm{~min}$, pre-UVO degrades the conductivity of MLG electrode, with the derived $R_{S}$ increased substantially to 66.7 $\Omega \mathrm{cm}^{2}$ at a treatment time of $8 \mathrm{~min}$, and a drop in $J_{S C}$ and FF is observed.

Devices with different Au thicknesses are also fabricated and characterized. Here, an optimal PCE at $2 \mathrm{~nm}$ is reached. While thinner Au may not suffice for fully modifying graphene surface, Au layer of larger thickness progressively reduces $J_{S C}$ since more incoming light is being blocked. We found that for Au layer under $2 \mathrm{~nm}$, the loss in transparency is usually within a reasonable value of $6 \%$. However, this loss goes up rapidly for $3 \mathrm{~nm}$ and beyond $(>10 \%)$. It should be noted that UVO treatment has negligible effect on the transparency of our MLG.

We also investigate WF of MLG samples with Au nanoclusters and UVO treatments. Their UPS spectra are shown in Fig. 2. WF is calculated from the energy difference between secondary electron cut-off and the Fermi level $\left(E_{F}\right){ }^{21}$ The WF of pristine MLG is found to be $4.4 \mathrm{eV}$. Evaporation of $\mathrm{Au}(2 \mathrm{~nm})$ on MLG has in fact reduced the WF to $4.1 \mathrm{eV}$ attributed to "push-back" effect. ${ }^{22}$ After treating identical sample with 2 min of post-UVO, the WF increases significantly to $4.8 \mathrm{eV}$, i.e., a $0.7 \mathrm{eV}$ upshift in $\mathrm{WF}$ is achieved which offers a much more favorable interfacial energy

TABLE I. Performance summary of MLG/Au PSCs with different preUVO time and Au evaporation/post-UVO of $2 \mathrm{~nm} / 2 \mathrm{~min}$.

\begin{tabular}{lccccc}
\hline \hline $\begin{array}{l}\text { Pre-UVO } \\
\text { time (min) }\end{array}$ & $\begin{array}{c}J_{S C} \\
\left(\mathrm{~mA} / \mathrm{cm}^{2}\right)\end{array}$ & $\begin{array}{c}V_{O C} \\
(\mathrm{~V})\end{array}$ & $\begin{array}{c}\mathrm{FF} \\
(\%)\end{array}$ & $\begin{array}{c}\mathrm{PCE} \\
(\%)\end{array}$ & $\begin{array}{c}R_{S} \\
\left(\Omega \mathrm{cm}^{2}\right)\end{array}$ \\
\hline 1 & 3.4 & 0.52 & 31.2 & 0.55 & 67.2 \\
2 & 3.9 & 0.51 & 32.0 & 0.64 & 63.6 \\
3.5 & 5.2 & 0.54 & 44.1 & 1.24 & 21.9 \\
5 & 4.3 & 0.52 & 42.6 & 0.95 & 50.5 \\
8 & 3.6 & 0.52 & 32.8 & 0.61 & 66.7 \\
\hline \hline
\end{tabular}




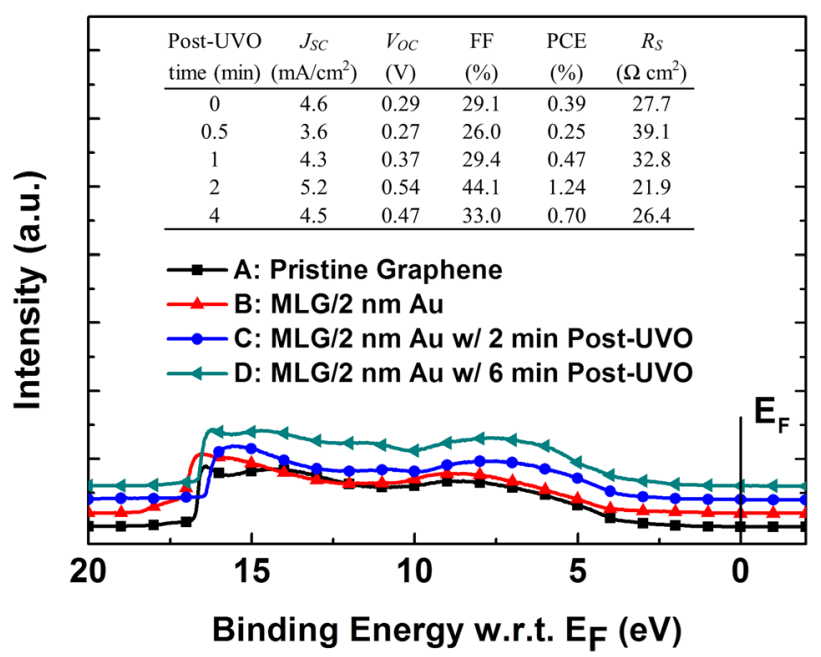

FIG. 2. (Color online) UPS spectra of different MLG samples. A: pristine MLG only, B: MLG/pre-UVO $(3.5 \mathrm{~min}) / \mathrm{Au}(2 \mathrm{~nm})$ without post-UVO, C: MLG/pre-UVO (3.5 min)/Au ( $2 \mathrm{~nm})$ with $2 \mathrm{~min}$ post-UVO, D: MLG/preUVO $(3.5 \mathrm{~min}) / \mathrm{Au}(2 \mathrm{~nm})$ with $6 \mathrm{~min}$ post-UVO. Inset shows the performance of MLG/Au PSCs with different post-UVO time and pre-UVO/Au evaporation of $3.5 \mathrm{~min} / 2 \mathrm{~nm}$.

matching with the highest occupied molecular orbital (HOMO) of P3HT $(\sim 5.0 \mathrm{eV})$ and is beneficial in two aspects: (i) enhanced $V_{O C}$ due to better ohmic contact formed and (ii) lowered $R_{S}$ due to the reduced injection barrier. The benefits owing to the proper post-UVO are confirmed by the MLG/Au PSCs with different durations of post-UVO as listed in the inset of Fig. 2. Indeed, from no treatment to $2 \mathrm{~min}$ of post-UVO, $V_{O C}$ increases notably from $0.29 \mathrm{~V}$ to $0.54 \mathrm{~V}$, while $R_{S}$ reduces from $27.7 \Omega \mathrm{cm}^{2}$ to $21.9 \Omega \mathrm{cm}^{2}$ with improved FF and $J_{S C}$. Nevertheless, excess post-UVO negatively affects the WF of MLG. As observed from UPS, the WF of MLG/Au sample drops from $4.8 \mathrm{eV}$ to $4.6 \mathrm{eV}$ after 6 min of post-UVO, which may account for the decreased $V_{O C}$ in device treated with 4 min of post-UVO. Besides lowering WF, excess post-UVO is also detrimental to the conductivity of graphene, resulting in performance degradation for post-UVO $\geq 4 \mathrm{~min}$.

While current MLG/Au devices have not yet reached competitive performance with PEDOT-based ITO device, there is clearly much room in making improvement from two notable aspects. In this work, the starting graphene for device study is undoped in order to fully investigate the effect of UVO-treated Au and is of multi-layer to ensure acceptable electrical conductivity, at the cost of performance to a certain extent. Furthermore, chemical doping has been introduced as an effective method to modify the properties of graphene, benefiting both the conductivity ${ }^{23}$ and WF. ${ }^{24}$ Therefore, possible approaches for future improvement of MLG/Au PSCs may arise from a combination of employing better fabrication technique, introducing doping to graphene samples, and improving existing method.

In summary, a simple yet effective method of modifying the interfacial properties of graphene by depositing a thin layer of Au assisted by separate UVO processes is proposed and demonstrated as an alternative to conventional PEDOT:PSS for realizing efficient graphene anodes. With proper UVO treatments on MLG/Au (2nm), PSCs with $V_{O C}$ of $0.52-0.54 \mathrm{~V}, J_{S C}$ of $4.5-5.5 \mathrm{~mA} / \mathrm{cm}^{2}$, FF of $43-48 \%$, and PCE up to $1.24 \%$ are obtained, exhibiting largely enhanced performance compared with MLG PSCs directly modified by PEDOT:PSS and UVO. With UPS characterization, the performance improvement can be understood from two aspects. First, $R_{S}$ is significantly reduced attributed to (i) favorable band alignment at the graphene/polymer interface, (ii) improving interfacial contact by Au layer, and (iii) short UVO durations. As a result, FF and $J_{S C}$ considerably increase. Second, injection barrier is reduced for better ohmic contact and thus a large $V_{O C}$. Our demonstration of improvement in performance of graphene PSCs can promote further research efforts on realizing efficient and flexible graphene PSCs as the next generation organic photovoltaic device.

This work is financially supported by UGC Grant Nos. 10400897 and 10401466 of the University of Hong Kong, the General Research Fund (HKU\#712010) from the Research Grants Council of Hong Kong Special Administrative Region, China, and Jiawei SolarChina, Co. Ltd. Zhang would like to acknowledge Hong Kong Ph.D. Fellowship from the Research Grants Council of Hong Kong. Zhu thanks the supports by National Science Foundation of China (50972067) and National Program on Key Basic Research Project (973 Program, 2011CB013000-G05).

${ }^{1}$ G. Dennler, M.C. Scharber, and C.J. Brabec, Adv. Mater. 21, 1323 (2009). ${ }^{2}$ Y. Wang, S. W. Tong, X. F. Xu, B. Özyilmaz, and K. P. Loh, Adv. Mater. 23, 1514 (2011).

${ }^{3}$ A. Schlatmann, Appl. Phys. Lett. 69, 1764 (1996).

${ }^{4}$ Z. Chen, B. Cotterell, W. Wang, E. Guenther, and S.-J. Chua, Thin Solid Films 394, 201 (2001).

${ }^{5}$ S. Pang, Y. Hernandez, X. Feng, and K. Müllen, Adv. Mater. 23, 2779 (2011).

${ }^{6}$ L. Gomez De Arco, Y. Zhang, C. W. Schlenker, K. Ryu, M. E. Thompson, and C. Zhou, ACS Nano 4, 2865 (2010).

${ }^{7}$ Y. Wang, X. Chen, Y. Zhong, F. Zhu, and K. P. Loh, Appl. Phys. Lett. 95, 063302 (2009).

${ }^{8}$ P. Hyesung, J. A. Rowehl, K. K. Kim, V. Bulovic, and J. Kong, Nanotechnology 21, 505204 (2010).

${ }^{9}$ M. Choe, B. H. Lee, G. Jo, J. Park, W. Park, S. Lee, W.-K. Hong, M.-J. Seong, Y. H. Kahng, K. Lee et al., Org. Electron. 11, 1864 (2010).

${ }^{10}$ Y.-H. Kim, S.-H. Lee, J. Noh, and S.-H. Han, Thin Solid Films 510, 305 (2006).

${ }^{11}$ J. van de Lagemaat, Appl. Phys. Lett. 88, 233503 (2006).

${ }^{12}$ J. Meyer, R. Khalandovsky, P. Görrn, and A. Kahn, Adv. Mater. 23, 70 (2011).

${ }^{13}$ C.-P. Chen, Y.-D. Chen, and S.-C. Chuang, Adv. Mater. 23, 3859 (2011).

${ }^{14}$ K. X. Steirer, J. P. Chesin, N. E. Widjonarko, J. J. Berry, A. Miedaner, D. S. Ginley, and D. C. Olson, Org. Electron. 11, 1414 (2010).

${ }^{15}$ Y.-Y. Lee, K.-H. Tu, C.-C. Yu, S.-S. Li, J.-Y. Hwang, C.-C. Lin, K.-H. Chen, L.-C. Chen, H.-L. Chen, and C.-W. Chen, ACS Nano 5, 6564 (2011).

${ }^{16}$ C.-D. Wang and W. C. H. Choy, Sol. Energy Mater. Sol. Cells 95, 904 (2011).

${ }^{17}$ X. Li, H. Zhu, K. Wang, A. Cao, J. Wei, C. Li, Y. Jia, Z. Li, X. Li, and D. Wu, Adv. Mater. 22, 2743 (2010).

${ }^{18}$ Z. Li, H. Zhu, D. Xie, K. Wang, A. Cao, J. Wei, X. Li, L. Fan, and D. Wu, Chem. Commun. 47, 3520 (2011).

${ }^{19} \mathrm{G}$. Li, V. Shrotriya, J. Huang, Y. Yao, T. Moriarty, K. Emery, and Y. Yang, Nature Mater. 4, 864 (2005).

${ }^{20}$ D. D. S. Fung, L. Qiao, W. C. H. Choy, C. Wang, W. E. I. Sha, F. Xie and S. He, J. Mater. Chem. 21, 16349 (2011).

${ }^{21}$ K. Fehse, J. Appl. Phys. 102, 073719 (2007).

${ }^{22}$ S. Braun, W. R. Salaneck, and M. Fahlman, Adv. Mater. 21, 1450 (2009).

${ }^{23}$ S. Bae, H. Kim, Y. Lee, X. Xu, J.-S. Park, Y. Zheng, J. Balakrishnan, T. Lei, H. R. Kim, Y. I. Song et al., Nat. Nanotechnol. 5, 574 (2010).

${ }^{24}$ Y. Shi, K. K. Kim, A. Reina, M. Hofmann, L.-J. Li, and J. Kong, ACS Nano 4, 2689 (2010). 